\title{
ЗАВИСИМОСТЬ НЕОДНОРОДНОГО УШИРЕНИЯ СПЕКТРОВ ЛЮМИНЕСЦЕНЦИИ ОТ КОНЦЕНТРАЦИИ МОЛЕКУЛ АНТРАЦЕНА В
}

\section{Введение}

Неоднородное уширение в спектрах люминесценции, характерное для всех примесей в твердотельных матрицах, отражает неоднородность распределения примесных центров в матрице $\left[{ }^{1,2}\right]$. Неоднородность матрицы можно в первом приближении выразить в виде функции неоднородного распределения примесных молекул по энергиям (0-0)-переходов $\left[3,{ }^{3}\right]$.

При лазерном возбуждении в резонансе с чисто электронным переходом удается снять значительную долю неоднородного уширения в спектре люминесценции $\left[{ }^{5,6}\right]$, что дает возможность изучать однородное уширение бесфононных линий, отражающее процессы преобразования энергии в примесной молекуле. С этой целью в последнее время был использован ряд новых методик, в частности выжигание провала в спектрах $\left[{ }^{7-10}\right]$, фотонное эхо $\left[{ }^{11}\right]$ и распад оптической свободной индукции $\left[{ }^{12}\right]$, а также проведены прямые измерения ширины чисто электронной линии при резонансном возбуждении (с использованием модуляции света) $\left[{ }^{13,14}\right]$. Однако интересно разобраться не только в однородном, но и в неоднородном уширении спектров люминесценции примесных молекул, в том числе и систем Шпольского с умеренным неоднородным уширением. В мультиплетной структуре спектров поглощения и люминесценции систем Шпольского проявляется дискретная природа функции неоднородного распределения примесных молекул в кристаллах, и ширины компонентов мультиплетов при гелиевых температурах составляют от нескольких десятых до одного $\mathrm{CM}^{-1}$. В $\left.{ }^{4}\right]$ для измерения упомянутой функции разработана методика двойного сканирования в системах с хорошо разрешенной вибронной структурой.

Одним из возможных способов исследования неоднородного уширения спектров является варьирование концентрации дефектов, вызывающих уширение $\left[{ }^{2}\right]$. При изменении числа примесных центров в системах Шпольского неоднородное уширение спектров действительно изменяется (см. обзор $\left.\left[{ }^{15}\right]\right)$. В $\left[{ }^{16}\right]$ было показано, что при повышении концентрации молекул бенз(а)пирена в $н$-октане и $H$-гептане полосы в спектре флуоресценции при $77 \mathrm{~K}$ становятся шире.

В данной работе исследуется влияние концентрации примесных молекул на неоднородное уширение спектров возбуждения и флуоресцен- 
ции систем Шпольского - антрацен в н-октане $(\mathrm{C}(8)-\mathrm{A})$ и антрацен в н-гексане (C (6) -A). *

Квазилинейчатые спектры антрацена в матрицах Шпольского изучались в $\left[{ }^{18-21}\right]$. Подробный анализ спектров поглощения и флуоресценции антрацена в $H$-парафинах при $4 \mathrm{~K}$ проведен в $\left[{ }^{19}\right] .(0-0)$-Мультиплет системы С (8)-A состоит из восьми линий, расположенных на участке шириной до $700 \mathrm{~cm}^{-1}$, а система $\mathrm{C}(6)-\mathrm{A}$ имеет дублетную структуру с разностью частот компонентов дублета в $\Delta v=163 \mathrm{~cm}^{-1}$ $\left[{ }^{19}\right]$ (компоненты $a$ и $c$ в нашем обозначении (см. таблицу)).

Частоты $v$ компснентов $(0-0)$-мультиплета при $4,2 \mathrm{~K}, \mathrm{~cm}^{-1}$

\begin{tabular}{|c|c|c|c|c|}
\hline \multicolumn{2}{|c|}{$\mathrm{C}(6)-\mathrm{A}$} & \multirow{3}{*}{ Линия } & \multicolumn{2}{|c|}{$C(8)-A$} \\
\hline \multirow[b]{2}{*}{ Линия } & \multirow[b]{2}{*}{$v, c M^{-1}$} & & \multicolumn{2}{|c|}{$v, C M^{-1}$} \\
\hline & & & $\begin{array}{c}\text { при } \\
c=10^{-6}, \\
\text { моль } / \Omega\end{array}$ & $\begin{array}{c}\text { при } \\
c=10^{-2}, \\
\text { моль } / \Omega\end{array}$ \\
\hline $\begin{array}{l}a \\
b \text { * } \\
c \\
d \\
e\end{array}$ & $\begin{array}{l}26493 \\
26450 \\
26330 \\
26163 \\
26129\end{array}$ & $\begin{array}{l}A \\
B \\
C \\
D \\
E \\
F \\
G \\
H\end{array}$ & $\begin{array}{c}26629 \\
26600 \\
26580 \\
26370 \\
26163 \\
- \\
-\end{array}$ & $\begin{array}{l}26629 \\
26600 \\
26580 \\
26337 \\
26163 \\
26105 \\
26014 \\
25926\end{array}$ \\
\hline
\end{tabular}

* Линия $b$ проявляется только в спектре возбуждения; в спектре флуоресценции она скрыта фононным крылом интенсивной бесфононной линии $a$.

** Полоса с полушириной $\sim 180 \mathrm{~cm}^{-1}$.

\section{Методика измерений}

Растворы антрацена в $\boldsymbol{H}$-гексане и $\boldsymbol{H}$-октане приготавливались с концентрацией $c$ от $10^{-7}$ до $10^{-3}$ моль/л при комнатной температуре. Растворение антрацена в $H$-парафинах до $c=10^{-2}$ моль/л проводилось при температуре $50^{\circ} \mathrm{C}$. Растворители марки «хч» были дополнительно очищены (пропущены через колонку с силикагелем и ректифицированы) **. Как известно $\left[{ }^{22}\right]$, неоднородная ширина бесфононных линий в спектрах систем Шпольского зависит от условий замораживания. Нами применен следующий способ: кюветы с растворами (объемом 0,2 см ${ }^{3}$ при толщине слоя $0,3-0,6$ мм) погружались в жидкий азот, а после кристаллизации раствора быстро перемещались в гелиевый криостат и опускались в жидкий гелий (влияние концентрации примеси на условия замораживания не учитывалось). Все спектры измерены при температуре кипения жидкого гелия $4,2 \mathrm{~K}$.

Спектры флуоресценции и возбуждения измерялись на автоматизированной спектрометрической системе в секторе спектроскопии кристаллов Института физики АН ЭССР $\left[{ }^{23}\right]$. Флуоресценция возбуждалась светом лампы ДКсШ-1000 через двойной монохроматор МДР-1 и регистрировалась с помощью охлаждаемого ФЭУ-106 в режиме счета фо-

* Известно, что при высоких концентрациях примесных молекул в процессе кристаллизации матрицы образуются молекулярные ассоциаты $[17,18]$, однако здесь эти вопросы не рассматриваются.

** Автор благодарен Л. Паальме за очнстку растворителей. 
тонов через двойной монохроматор ДФС-12 с накоплением в многоканальном анализаторе NTA-512B, соединенном с ЭВМ «Наири-2». Для учета искажающего действия спектрального прибора при измерении ширины бесфононных линий предварительно измерялась аппаратная функция монохроматора ДФС-12, которая хорошо аппроксимируется гауссовой кривой. Бесфононные линии в спектрах люминесценции систем Шпольского при гелиевых температурах имеют, по данным [ $\left.{ }^{22,24}\right]$, форму, также близкую к гауссовой, так что ширина измеряемой линии $\Gamma$ выражается как $\Gamma^{2}=\delta_{\text {aп }}^{2}+\delta^{2}$, где $\delta_{\text {aп }}$ - полуширина аппаратной функции, $\delta$ - полуширина бесфононной линии. Ошибка измерений ширины бесфононных линий $\pm 0,5 \mathrm{~cm}^{-1}$.

\section{Результаты измерений и обсуждение}

Методом селективного возбуждения отдельных компонентов мультиплета Шпольского $\left[{ }^{25,26}\right]$ измерены частоты $(0-0)$-линий, проявляющиеся в спектрах флуоресценции и возбуждения систем С (6) - А и C(8)-A (см. таблицу). Наши данные о мультиплетной структуре системы С (8)-A при высоких концентрациях примеси $(c \geqslant$ $\geqslant 3 \cdot 10^{-4}$ моль/ $\left./ \Omega\right)$, а также о наличии в системе $\mathrm{C}(6)$ - А двух интенсивных компонентов подтвердили результаты $\left[{ }^{19}\right]$. Однако, кроме этих компонентов, нами обнаружены и измерены еще три слабые $(0-0)$-линии $b, d, e$ (с их колебательными повторениями), интенсивность кото-

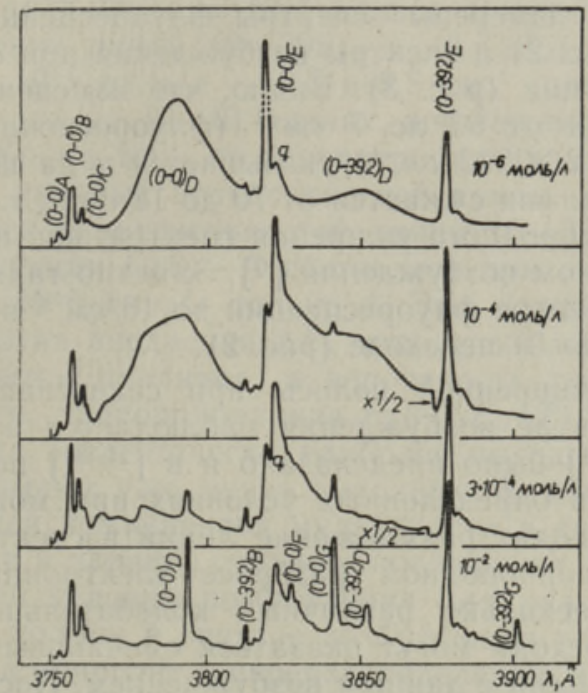

Рис. 1. Участок спектра флуоресценции системы С (8)-A при различных концентрациях молекул антрацена. $T=4,2 \mathrm{~K}$, длина волны возбуждаюшего света $\lambda_{\text {возб }}=$ $=3560 \AA$, ширина возбуждения $\delta_{\text {возб }}=$ $=160 \mathrm{~cm}^{-1}$. Обозначения: $A, B \ldots-$ компоненты мультиплета; $\quad(0-0)_{B}^{\cdots}-$ $(0-0)$-линия компонента $B ;(0-392)_{B}-$ колебательное повторение $v_{00}-392 \mathrm{~cm}^{-1}$ компонента $B ; \bar{q}$ - максимумы фононных крыльев.

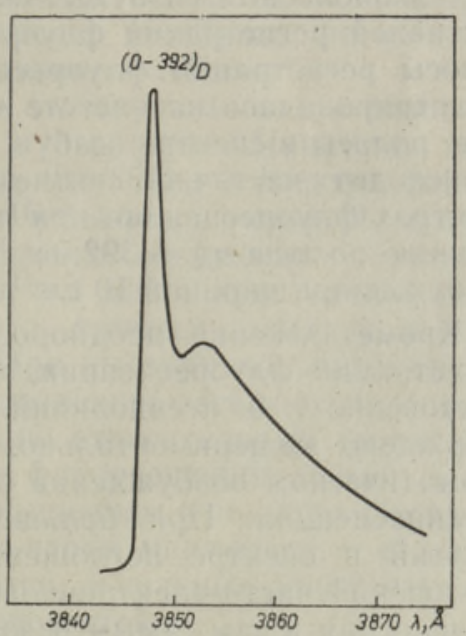

Рис. 2. Участок перехода $v_{00}-$ - $392 \mathrm{~cm}^{-1}$ в спектре флуоресценции системы $\mathrm{C}(8)-\AA$ при концентрации антрацена $10^{-4}$ моль/л. $T=4,2 \mathrm{~K}$, возбуждение в области $(0-0)$-перехода, ширина возбуждения $\delta_{\text {возб }}=$ $=10 \mathrm{~cm}^{-1}$. Ширнна $(0-392)$ линии $\delta=16 \mathrm{CM}^{-1}$. 


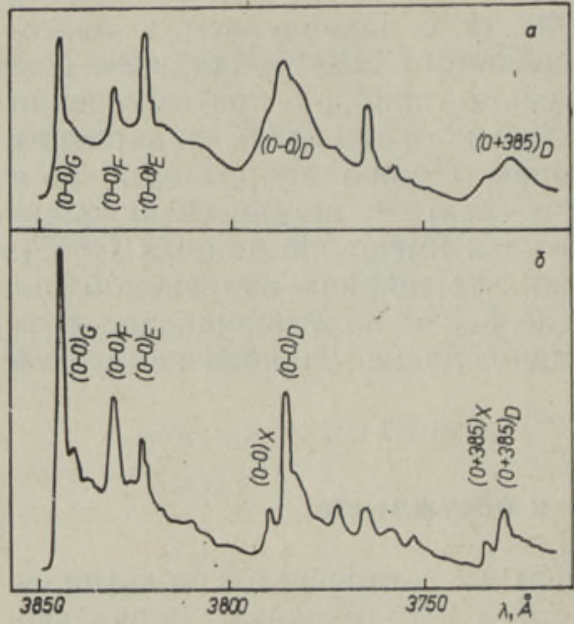

Рис. 3. Участок спектра возбуждения системы С (8) - А при концентрации антрацена $10^{-4}$ моль/л. $T=4,2 \mathrm{~K}$, длина волны регистрации флуоресценции $\lambda_{\text {per }}=$ $=3902 \AA$, ширина регистрации флуоресценции $\Delta v_{\text {per }} 66$ (a) и $7 \mathrm{cM}^{-1}$ (б). $D, E, \ldots$ - компоненты мультиплета. Наблюдается возникновение псевдолиний $(0-0)_{x}$ и $(0+385) x$.

рых оказалась на два порядка слабее интенсивности компонентов $a$ и $c$.

Область чисто электронного перехода $S_{1} \rightarrow S_{0}$ и его колебательного повторения $v_{00}-392 \mathrm{~cm}^{-1}$ (частоты локальных колебаний молекулы антрацена, определенные по разным компонентам мультиплетов, различаются до $4 \mathrm{~cm}^{-1}$ ) в спектре флуоресценции С (8)-А при разных концентрациях примесных молекул изображена на рис. 1. При низких концентрациях примеси $\left(c \leqslant 10^{-4}\right.$ моль/л) наряду с квазилиниями присутствует широкая полоса с полушириной $\sim 180 \mathrm{~cm}^{-1}$ и максимумом при $\sim 3790 \AA$. Эта полоса повторяется по всему спектру с частотами локальных колебаний молекулы антрацена. Для выяснения вопроса о прсисхождении широких полос (уширение однородное или неоднородное) были измерены спектры флуоресценции при узкополосном возбуждении (рис. 2) и спектры возбуждения при селективной регистрации флуоресценции (рис. 3). Видно, что изменение полосы регистрации флуоресценции от 66 до $7 \mathrm{~cm}^{-1}$ (флуоресценцня регистрировалась на частоте $v_{00}-2 \times 392$ см $^{-1}$ ) сказывается и на ширине полосы в спектре возбуждения: она сужается от 70 до $12 \mathrm{~cm}^{-1}$, т. е. происходит частичное снятие неоднородного уширения спектра, как и в спектрах флуоресценции при лазерном возбужденни $\left[{ }^{6}\right]$. Заметно также сужение полосы $v_{00}-392 \mathrm{~cm}^{-1}$ в спектре флуоресценции до $16 \mathrm{~cm}^{-1}$ при возбуждении шириной $10 \mathrm{~cm}^{-1}$ на $(0-0)$-переходе (рис. 2).

Кроме сужения неоднородно уширенной полосы при селективной регистрации флуоресценции, в спектре возбуждения наблюдается возникновение т. н. псевдолиний. В $\left[{ }^{3}\right]$ было предсказано и в $\left[{ }^{27-30}\right]$ подтверждено экспериментально, что в определенных условиях при монохроматическом возбуждении происходит размножение линий в спектре люминесценции. При большом неоднородном разбросе электронных энергий в спектре поглощения несколько различных колебательных повторений чисто электронного перехода могут оказаться одинаковыми по энергиям и тем самым резонансными с данным возбуждением. После колебательной релаксации это приводит к возникновению в спектре нескольких различающихся по частоте $(0-0)$-линий (и их повторений по локальным колебаниям), отстоящих от монохроматического возбуждения на величину частоты локальных колебаний примесной молекулы в возбужденном -электронном состоянии.

Дублет в спектре возбуждения системы С $(8)-\mathrm{A}\left((0-0)_{X}\right.$ и $(0-0)_{D}$ на рис. 3), возникающий при селективной регистрации, обусловлен наличнем двух частот колебаний в основном электронном состоянии молекулы антрацена (760 и $\left.2 \times 392 \mathrm{~cm}^{-1}\right)$ в пределах величины неоднородного ушнрения. Колебательные уровни с частотами 760 и $2 \times 392$ см $^{-1}$ 
оказываются в резонансе с частотой регистрации флуоресценции $v_{\text {per }}=$ $=25618 \mathrm{~cm}^{-1}$. Расстояние от частоты регистрации флуоресценции $v_{\text {per }}$ до частот линий дублета $\left(v(0-0)_{X}=26375 \mathrm{~cm}^{-1}, \quad v(0-0)_{D}=\right.$ $\left.=26405 \mathrm{~cm}^{-1}\right)$ совпадает с частотами локального колебания $760 \mathrm{~cm}^{-1}$ и второго обертона локального колебания $392 \mathrm{~cm}^{-1}$ в основном электронном состоянии молекулы антрацена.

Рассмотрим зависимость спектров флуоресценции от концентрации примесных молекул в системе С (8)-A. При $c=3 \cdot 10^{-4}$ моль/ $\Omega$ и выше спектр флуоресценции существенно изменяется - широкая полоса с полушириной $180 \mathrm{~cm}^{-1}$ и максимумом при $3791 \AA$ переходит в квазилинию $3796 \AA$ с полушириной $8,5 \mathrm{~cm}^{-1}$ (см. рис. 1). Подобное сужение наблюдается и в спектре возбуждения. Таким образом, при повышении концентрации примесных молекул частично снимается неоднородное уширение спектров флуоресценции и возбуждения, т. е. существенно изменяется вид функции неоднородного распределения примесей. При $c \geqslant 3 \cdot 10^{-4}$ моль/ $\Omega$ число компонентов мультиплета увеличивается. При низких концентрациях часть $(0-0)$-линий системы С $(8)-A$, видимо, скрыта фоном повторения локального колебания $v_{00}-392 \mathrm{~cm}^{-1}$ неоднородно уширенной полосы $D$.

Аналогичное превращение полосатых спектров флуоресценции в квазилинейчатые при повышении концентрации примесей наблюдалось ранее для некоторых ароматических молекул в поликристаллических матрицах при $T=77 \mathrm{~K}$ (см. обзор $\left.\left[{ }^{15}\right]\right)$. Однако в нашем случае весьма чувствителен к изменению концентрации лишь один тип центров. Например, неоднородная ширина линии $(0-392)_{E}$ в спектре флуоресценции системы $\mathrm{C}(8)-\AA$ изменяется от $6,7 \mathrm{~cm}^{-1}$ при $c=10^{-7}$ моль/л до $8,2 c \mu^{-1}$ при $c=10^{-2}$ моль/ . В системе $\mathrm{C}(6)-\mathrm{A}$ при $c=10^{-6}$ моль/ $/ \Omega$ ширина линий $(0-395)_{a}$ и $(0-395)_{c}$ составляет 5,9 и $7,3 \mathrm{~cm}^{-1}$, а при $c=10^{-2}$ моль/ $/ \Omega-7,5$ и $8,5 \mathrm{~cm}^{-1}$ соответственно (возбуждение во всех случаях на $(0-0)$-линиях, ширина возбуждения $\left.\sim 100 \mathrm{~cm}^{-1}\right)$.

Кроме того, от концентрации примесных молекул зависит и отношение пиковых интенсивностей компонентов мультиплета. Например, отношение интенсивностей линий $(0-392)_{E}$ и $(0-392)_{G}$ в спектре флуоресценции $\mathrm{C}(8)-\mathrm{A}$ составляет при $c=3 \cdot 10^{-4}$ моль/ $/$ около 14 , а при $c=10^{-2}$ моль/л только 4. Это можно объяснить следующим образом. Энергия внедрения $\Delta E_{s}$ примесных молекул в $s$ места кристаллической решетки различна, а вероятность того, что примеси попадут в эти места, пропорциональна $\exp \left(\Delta E_{s} / k T\right)$ ***. При высоких концентрациях, когда энергетически наиболее выгодные положения заселены, примеси занимают уже менее выгодные. К изменению относительных интенсивностей компонентов мультиплета в спектре флуоресценции может привести и влияние сильного поглощения. С повышением концентрации пріімесей условия возбуждения для разных центров меняются, т. е. слабые полосы в спектре возбуждения усиляются, а сильные ослабляются, что и приводит к перераспределению интенсивностей.

Механизмы превращения неоднородно уширенной полосы в квазилинию в спектрах флуоресценции и возбуждения системы С (8)-А при повышении концентрации примесных молекул нам представляются следующими. В системах с низкой концентрацией примеси часть молекул антрацена попадает в несколько различные условия в поликристаллической матрице $н$-октана, в результате чего вибронные уровни примесей сильно возмущаются со стороны матрицы, вызывая значительное

ж** Имеется в виду равновесное распределение, которое в самом деле не достигается; энтропийный числитель здесь не учитывается. 
неоднородное уширение ****. Возможны две причины уменьшения неоднородного уширения спектров при повышении концентрации.

1. При низких концентрациях имеется сильно неоднородный набор центров $D$, которые находятся в энергетически более выгодных позициях в матрице. С повышением концентрации примесей (антрацена) некоторые центры (обозначим их через $D^{\prime}$ ) из-за нехватки выгодных мест вынуждены занять места, энергетически менее выгодные, но обладающие меньшим неоднородным уширением. Таким $D^{\prime}$-центрам в спектрах возбуждения и флуоресценции соответствует квазилиния при $3796 \AA$ А. В то же время относительная интенсивность полос в спектрах уменьшается. Ослабление неоднородно уширенной полосы может быть обусловлено образованием агрегатов антрацена, в которые объединяются центры $D$, ответственные за эту полосу $\left[{ }^{32}\right]$. В результате в спектрах появляется квазилиния, а широкая полоса сильно ослабляется, т. е. при высоких концентрациях доминируют существенно иные неоднородные центры $D^{\prime}$.

2. Изучением колебательной структуры спектров (фононные крылья) молекулярных кристаллов, а также исследованиями ядерного квадрупольного резонанса [ $\left.{ }^{33}\right]$ установлено, что примеси, внедренные в кристаллическую решетку, приводят к ее искажению. Величина искажения зависит от размеров и концентрации примесных молекул, а также от условий кристаллизации. Повышенные концентрации примесных молекул вызывают локальное изменение структуры кристаллической матрицы $\left[{ }^{34,15}\right]$, в результате чего в спектрах возбуждения и флуоресценции изменяется неоднородное окружение центров $D$, и полосы, за которые они ответственны, сужаются. Однако вопрос - почему изменяется неоднородное окружение только у одного набора центров? остается открытым.

\section{Выводы}

При исследовании концентрационной зависимости спектров люминесценции примесных центров кристаллов проявляются различные эффекты их неоднородного уширения.

Измерение концентрационного ряда спектров возбуждения и флуоресценции антрацена в $\boldsymbol{H}$-гексане при $T=4,2 \mathrm{~K}$ показало, что в интервале концентраций от $10^{-6}$ до $10^{-2}$ моль/л линейчатая структура спектров полностью сохраняется. В системе антрацен в типа центров (центров $D$ ) обнаружена сильная и на первый взгляд неожиданная зависимость неоднородного уширения спектров возбуждения и флуоресценции от концентрации примеси - с повышением ее широкая полоса превращается в квазилинию. Другие компоненты мультиплета системы антрацен в н-октане в интервале концентраций от $10^{-7}$ до $10^{-2}$ моль/л сохраняют квазилинейчатый характер.

Автор глубоко благодарен К. Ребане за руководство работой, а также Т. Тамму и П. Саари за ряд ценных замечаний.

***** В системах Шпольского некоторая доля молекул встранвается в матрицу хаотически, обусловливая квазинепрерывное неоднородное распределение и слабый фон в спектре люминесценции [ $\left.{ }^{31}\right]$.

\section{Л И ТЕРА Т У Р А}

1. Р еб ане К. К. Элементарная теория колебательной структуры спектров примесных центров кристаллов. М., 1968.

2. Stoneham, A. M. Shapes of inhomogeneously broadened resonance lines in solids. - Rev. Mod. Phys., 1969, v. 41, N 1, p. 82-108. 
3. Ав арма 2 Р. Влияние монохроматического возбуждения на ширину и интенсивность неоднородно уширенных линий в спектре люминесценции. - Изв. АН ЭССР, Физ. Матем., 1974 , т. 23 , № 3, с. $238-247$.

4. Т а м м Т. Б., К и к а с Я. В., С и р к А. Э. Измерение функции неоднородного распределения примесных центров методом двойного сканирования спектров. ЖПС, 1976 , т. 24 , № 2, с. $315-321$.

T a m m, T., Kikas, J. Double-scanning technique for measuring statistical distribution of impurity molecules. - Report at the 3rd Annual Meeting of Federation of Analytical Chemistry and Spectroscopy Societies, 15-19 Nov. 1976, Philadelphia, ÚSA.

$5 \mathrm{Sz}$ a b o, A. Laser-induced fluorescence-line narrowing in ruby. - Phys. Rev. Lett., 1970 , v. 25 , N 14 , p. $924-926$.

6. П ерсонов Р. И., Альшиц Е. И., Быковск ая Л. А. Возникновение тонкой структуры в спектрах флуоресценции сложных молекул при лазерном возбуждении. - Письма в ЖЭТФ, 1972, т. 15, № 10, с. 609-612.

Персонов Р. И., Альшиц Е. И., Б ыковская.Л. А., Х арламов Б. М. Тонкая структура спектров люминесценции органических молекул при лазерном возбуждении и природа широких спектральных полос твердых растворов. - ЖЭТФ, 1973 , т. 65 , № 5, с. $1825-1836$.

7. Гороховски й А. А., К а арли Р. Қ., Ребане Л. А. Выжигание провала в контуре чисто электронной линии в системах Шпольского. - Письма в ЖЭТФ, 1974 , т. 20 , № 7 , с. $474-479$.

Gorokhovski, A. A., Ka a rli, R. K., Rebane, L. A. The homogeneous, pure electronic linewidth in the spectrum of a $\mathrm{H}_{2}$-phthalocyanine solution in $n$ octane at $5 \mathrm{~K}$ - - Optics Commun., 1976, v. 16, N 2, p. 282-284.

Gorokhovski, A. A., Rebane, L. A. The temperature broadening of purely electronic lines by the hole burning technique. - Optics Commun., 1977, v. $20, \mathrm{~N} 1, \mathrm{p} .144-146$.

8. Kharlamov, B. M., Personov, R. I., Bykovskaja, L. A. Stable «gap» in absorption spectra of solid solutions of organic molecules by laser irradiation. - Optics Commun., 1974, v. 12, N 2, p. 191-193.

9. De Vries, H., Wiersma, D. A. Homogeneous broadening of optical transitions in organic mixed crystals. - Phys. Rev. Lett., 1976, v. 36, N 2, p. 91-94.

10. Völker, S., van der Wa a Is, I. H. Laser-induced photochemical isomerization of free base porphyrin in a n-octane crystal at 4,2 K. - Molec. Phys., 1976, v. $32, \mathrm{~N} 6$, p. $1703-1718$.

11. A a r tsma, T. J., Wiersina, D. A. Photon-echo spectroscopy of organic mixed crystals. - Phys. Rev. Lett., 1976, v. 36, N 23, p. 1360-1362.

Photon-echo relaxation in molecular mixed crystals. - Chem. Phys. Lett, 1976 , v. 42 , N 3, p. $520-524$.

12. Gen ack, A. Z., Ma c farlane, R. M., Brewer, R. G. Optical free-induction decay in $\mathrm{LaF}_{3}: \mathrm{Pr}^{3+}$. - Phys. Rev. Lett., 1976, v. 37, N 16, p. 1078-1080.

13. Erickson, L. E. Fluorescence line narrowing of trivalent praseodymium in lanthanum trifluoride - the resonant transitions. - Optics Commun., 1975, v. $15, \mathrm{~N} 2$, p. $246-249$.

14. Delsart, C., Pelletier - Allard, N., Pelletier, R. Hyperfine structure of the $6011 \AA$ line of $\mathrm{Pr}^{3+}: \mathrm{LaCl}_{3}$ using the fluorescence line narrowing technique. - J. Phys. B: Atom. Molec. Phys., 1975, v. 8, N 17, p. 2771-2778.

15. Shpolskii, E. V., Bolotnikova, T. N., Modern trends in quasi-linear spectra studies. - Pure and Appl. Chem., 1974, v. 37, N 1-2, p. 183-195.

16. П ах апилль Ю. Квазилинейчатые спектры люминесценции пленок 3,4-бензпирена в $r$-парафинах при 77 К. - Изв. АН ЭССР, Физ. Матем., 1975, т. 24 , № 3 , с. $346-348$. 17. Гляд дов в к и й В. И., Клим ов а Л. А., Н е р се со в а Г. Н. Спектроскопия
смесей ароматических углеводородов в замороженных кристаллических раствоpax. II. Исследование характера агрегатов антрацена в $н$-парафинах. - Oпт. и спектр., 1967, т. 23, № 3, с. 407-413.

Климов а Л. А., Нерсес ова Г. Н., Глядковский В. И. Проявление локальных состояний агрегатных образований прнмеси в поликристаллических растворах. - Oпт, и спектр., 1976, т. 40, № 3, с. 500-504.

18. Болотникова Т. Н., Климова Л. А., Нерсесова Г. Н., Уткина Л. Ф. Исследование квазилинейчатых спектров флуоресценции и поглощения антрацена при 77,3 и 4,3К. - Опт. и спектр., 1966, т. 21 , № 4, с. $420-425$.

19. Macnab, R. M., S a uer, K. Electronic absorption and fluorescence spectra of anthracene, $\left[d_{10}\right]$-anthracene, and acridine in $n$-alkane matrices at $4 \mathrm{~K}$. J. Chem. Phys,, 1970, v. 53 , N 7, p. 2805-2817.

20. Richards, J. L., Rice, S. A. Study of impurity-host coupling in Shpolskii matrices. - J. Chem. Phys., 1971, v. 54, N 5, p. 2014-2023. 
21. Ferguson, J., Ma u, A. W.-H. Phosphorescence of anthracene. - Molec. Phys., 1974 , v. 28 , N 2, p. $469-477$.

22. Персонов Р. И., Годя ев Э. Д., Коротаев О. Н. О форме линий в квазилинейчатых спектрах люминесценции органических молекул при 4,2 К. - ФТТ, 1971, т. 13, № 1, с. 111-116.

23. С а а ри П. М., Кони Ю. Я., Пентярв Э. А., Си р К А. Э. Использование «Наири»-2» как он-лайн машины в экспериментальной установке. - Тезисы докл. Всесоюз. науч.-техн. конф. пользователей семейства ЭВМ типа «Наири», Харьков, 1975 , с. $223-227$.

24. Альшиц Е. И., Годяев Э. Д., Персонов Р. И. Уширение, форма и сдвиг бесфононных линий в спектрах примесных кристаллов н-парафинов в области температур $4,2-77$ K. - ФТТ, 1972 , т. 14, № 6, с. 1605-1612.

25. С в и щ в Г. М. К вопросу о природе тонкой структуры квазилинейчатых спектров ароматических углеводородов в замороженных парафиновых растворах. Изв. АН СССР, Сер. физ., 1963, т. 27, № 5, с. 696-699.

26. Р еб ан е К., С а а р и П., Т а м м Т. Природа «мультиплетов» и фононное крыло в спектрах некоторых систем Шпольского. - Изв. АН ЭССР, Физ. Матем., 1970 , т. 19 , № 2, c. $251-254$.

27. Ребане К. К., А в а м а а Р. А., Гороховский А. А. Структура бесфононных линий в неоднородно уширенных спектрах люминесценции при монохроматическом возбуждении. - Изв. АН СССР, Сер. физ., 1975, т. 39, № 9, c. $1793-1800$.

А в а р м а а Р. А., Го рох о в к и й А. А. Возникновение двойной структуры в неоднородно уширенных спектрах люминесценции. Молекула тетрацена в н-парафинах. - Опт. и спектр., 1975, т. 39 , № 2, с. 266-273.

28. Альшиц Е. И., Пер сонов Р. И., Стогов В. И. Зависимость спектров флуоресценции органических молекул в твердых растворах от длины волны лазерного излучения. - Изв. АН СССР, Сер. физ., 1975, т. 39, № 9, с. 1918-1921. А льши ц. И., П ер сонов Р. И., Пындик А. М., С тогов В. И. Зависимость спектров флуоресценции органических молекул в твердых растворах от длины волны лазерного возбуждения. - Опт. и спектр., 1975, т. 39, № 2, c. $274-280$.

29. Eberly, J. H., McColgin, W. C., Kawaoka, K., Marchetti, A. P. Probing broad lines in molecular spectra by optical site selection spectroscopy. Nature, 1974 , v. 251 , N 5472, p. 215-217.

30. Abram, I. I., Auerbach, R. A., Birge, R. R., Kohler, B. E., Steven : son, J. M. Narrow-line fluorescence spectra of perylene as a function of excitation wavelength. - J. Chem. Phys., 1975, v. 63, N 6, p. 2473-2478.

31. Р еб ан е К. К., Т а м м Т. Б. Вторичное свечение молекул перилена в матрице нормального гексана. - Тезисы докл. ХI Европ. конгресса по молекулярной спектроскопии, Таллин, № 172 (В5), 1973.

$\mathrm{T}$ a mm, T. On the drastic effects of inhomogeneity in Shpolsky system spectra. - ENSV TA Toim., Füüs. Matem., 1977, v. 26, N 1, p. $72-78$.

32. Шпольский Э. В., Климова Л. А., Нерсесова Г. Н., Глядков с к и й В. И. Концентрационная зависимость спектров флуоресценции и поглощения замороженных парафиновых растворов нафталина. - Опт. и спектр., 1968 , т. 24 , № 1, c. $52-59$.

33. Китайгородский А. И. Молекулярные кристаллы. М., 1971, с. 141-149.

34. Климова Л. А., Оглоблина А. И., Шпольский Э. В. К вопросу о природе зависимости резкости спектров от концентрации. - Изв. АН СССР, Сер. физ., 1970, т. 34, № 6, с. $1361-1364$.

Институт химии
Академии наук Эстонской ССР

Поступила в редакцию 29/VI 1977

\section{J. PAHAPILL}

\section{LUMINESTSENTSISPEKTRITE MITTEHOMOGEENSE LAIENEMISE SOLTUVUS ANTRATSEENI MOLEKULIDE KONTSENTRATSIOONIST $n$-PARAFIINIDE MAATRIKSITES}

Artiklis on esitatud Śpolski süsteemide antratseen- $n$-heksaan $(C(6)-A)$ ja antratseen-n-oktaan (C(8)-A) ergastus- ja fluorestsentsispektrite mốtmise tulemused temperatuuril $4,2 \mathrm{~K}$ lisandimolekulide kontsentratsiooni vahemikus $10^{-7}-10^{-2}$ mooli/1. Süsteemis $\mathrm{C}(8)-\mathrm{A}$ ilmneb nimetatud spektrite mittehomogeense laienemise omapärane 
sōltuvus kontsentratsioonist. Kui lisandi kontsentratsioon on madal $\left(c \leqslant 10^{-4}\right.$ mooli/1), koosnevad $(0-0)$-multiplett ja tema vônkekordused kvaasijoontest ning mittehomogeeilselt laienenud ribast (poollaius $\left.\sim 180 \mathrm{~cm}^{-1}\right)$. Kontsentratsiooni tôstes $\left(c \geqslant 3 \cdot 10^{-4}\right.$ mooli/1) ilmub. mittehomogeenselt laienenud riba asemele kvaasijoon poollaiusega $8,5 \mathrm{~cm}^{-1}$. Süsteemi C (8) - A multipleti teiste komponentide ja süsteemi C (6) - A foononivabade joonte mittehomogeenne laius sõltub lisandimolekulide kontsentratsioonist tunduvalt vähem. Kontsentratsiooni tõustes $10^{-7}$ kuni $10^{-2}$ mooli/l laienevad foononivabad (0-392)-jooned $2 \mathrm{~cm}^{-1}$ võrra.

\section{J. PAHAPILL}

\section{DEPENDENCE OF INHOMOGENEOUS BROADENING OF THE LUMINESCENCE SPECTRA OF ANTHRACENE MOLECULE CONCENTRATIONS IN $n$-PARAFFIN MATRICES}

In this paper the results of measuring the excitation and fluorescence spectra at different concentrations of impurity molecules between $10^{-7}$ and $10^{-2}$ mole/ 1 in Shpolsky systems anthracene in $n$-hexane $(C(6)-A)$ and $n$-octane $(C(8)-A)$ at temperature $4.2 \mathrm{~K}$ are reported. There appears a strong and peculiar concentration dependence of the inhomogeneous broadening of the excitation and fluorescence spectra in system $\mathrm{C}(8)-\mathrm{A}$. At low impurity concentration $\left(c \leqslant 10^{-4} \mathrm{~mole} / 1\right)$, besides quasilines, there is an inhomogeneously broadened band (bandwidth $\sim 180 \mathrm{~cm}^{-1}$ ) in $(0-0)$ and vibronic multiplets of the spectra. As the concentration increases $\left(c \geqslant 3 \cdot 10^{-4}\right.$ mole/1), the broad band turns into a quasiline with a halfwidth of $8.5 \mathrm{~cm}^{-1}$. The inhomogeneous linewidths of other multiplet components of the system $\mathrm{C}(8)-\mathrm{A}$ and the no-phonon lines in spectra of the system $\mathrm{C}(6)-\mathrm{A}$ are less sensitive to the variation of concentration. As the concentration of anthracene molecules in hosts increases from $10^{-7}$ to $10^{-2}$ mole/l, the halfwidth of the no-phonon $(0-392)$-lines increases by $\sim 2 \mathrm{~cm}^{-1}$. 